\title{
DEVELOPMENT OF BASIC CONCEPT OF ICT PLATFORMS DEPLOYMENT STRATEGY FOR SOCIAL MEDIA MARKETING CONSIDERING TECTONIC THEORY
}

\author{
Ivan Demydov ${ }^{1}$ \\ ivan.v.demydov@lpnu.ua
}

Najm Ahmad Baydoun ${ }^{1}$

najem.b@iptpowertech.com

Mykola Beshley ${ }^{1}$

beshlebmi@gmail.com

Mykhailo Klymash

mykhailo.m.klymash@lpnu.ua

Oleksiy Panchenko ${ }^{1}$

oleksij@gmail.com

${ }^{1}$ Department of Telecommunications

Lviv Polytechnic National University,

12 S. Bandery str., Lviv, Ukraine, 79013

\begin{abstract}
This paper presents authors analytical view on social impacts as targeted advertisement into the network environment using Omori tectonic theory for description the processes of audience response evolution. This could be extremely important and useful in the modern world to realize desirable e-Gov informational policy in the circumstances of hybrid treats emergence that is especially relevant for the informational space and reaching a cyber-supremacy. Some mathematical and algorithmic basics were contributed for narrative description of information and communications technologies (ICT) architectural deployment could be used for outer regulation of audience response character by Social Media Marketing (SMM) principles. That could be performed by controlled distribution of specified digital content that contains respective key phrases, for example social advertisements and analyzing respective feed-backs. Some results of the empiric study of live audience response dependence on controlled impacts are discussed. Election processes data and recent media recordings for preliminary proof of the contributed concept feasibility have been analyzed. There were shown using gathered empiric data sets, that the extent of impacts to targeted audience response intensity could be the subject of outer regulation. The index has been contributed for assessment the efficiency of the impact's propagation inside the audience by calculation of row correlation of keyword occurrence and audience response intensity. The approaches suggested in the article can be useful both for building effective interactive systems of state-society interaction and for detecting manipulative traits when influencing a specific audience.
\end{abstract}

Keywords: controlled social impact, SMM, ICT, targeted content advertising, e-Gov strategy, cyberspace, tectonic theory.

DOI: $10.21303 / 2461-4262.2020 .001101$

\section{Introduction}

Many of us heard about earthquakes and their forecasting. Current models for stresses forecasting are not precise, due to long term character of earthquakes recurrence and relatively low volumes of statistics. But, some of them are mathematically suitable to describe the distribution of the information activity peaks intensity in the blogosphere [1], especially while applying SMM procedures actively. Other works, such as [2, 3], investigate the distribution of message lengths and dependence of its parameters on informational events excitation and excitation wave propagation in a homogeneous scale-free network features respectively. The distribution of the information activity peaks intensity turned out to be similar to the distribution of the intensity of underground shocks in the earth's crust - aftershocks (small stresses following the main stresses), the number of aftershocks decreases inversely in proportion to the time elapsed since the moment of the main 
stress, and this law is the name of the Japanese scientist Omori, which at the end of the nineteenth century observed aftershocks of a strong earthquake in central Japan and foreshocks (small shocks preceding the main blow) [4].

In [1] two types of information shocks were distinguished, the "seismograms" of which differ radically. A graph similar to a bell for an endogenous (internal) stress present a peak preceded by a curve that fixes the gradual increase in the frequency of informational events (i. e. targeted informational activities intensity), followed by a near-symmetrical form of attenuation. The moments of stresses it fixes are induced by information circulating in the blogosphere of informational space for a long time, both before and after the peak. An example of the endogenous event is elected President's inauguration after final declaration of the poll.

The authors of the article also point out that the peak and post-peak reaction of the informational space correlates with the behavior of key word markers preceding an endogenous shake, that is, in a certain sense, predictable. Hence the hypothesis is reasonable that knowing the behavior of precursors can even control this reaction, to build a predictable SMM automated system. As of 2011, by the keywords there were revealed about 150 of individual endogenous events, but exogenous (that is, caused from the outside) - about 1000 [1]. In fact, it was shown that exogenous events are an order of magnitude greater than endogenous, and one media event generally corresponds to events in several word frequencies (occurrences) of related keywords.

Obviously, the blogosphere, while being a part of the information space, is at the same time a highly-strung environment that loses equilibrium in two main scenarios. According to the first one, endogenous (Fig. 1, b), in part of this medium, the gradual ordering of information begins (participants in the information environment, bloggers show increasing interest in a particular topic), reaching at some point the least entropy (the maximum of discussion), followed by a gradual relaxation and loss of information (interest in the subject of information impact disappears). This is an oscillating process, and it is important that the information involved in it is contained in the oscillating system itself, that is, endogenous.

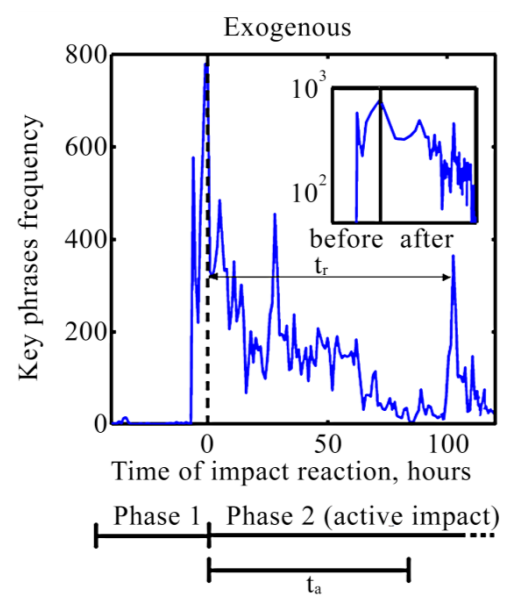

$a$

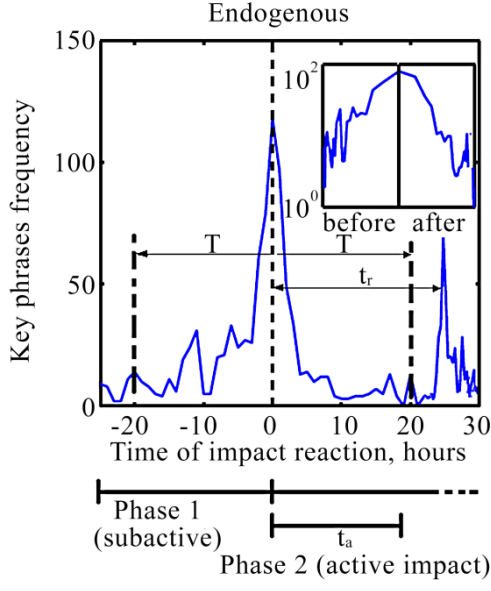

$b$

Fig. 1. The two basic scenarios of impact evolution through informational events into cyberspace: $a$ - exogenous; $b$ - endogenous (by [1], modified by authors)

The second, exogenous, scenario is quite different: despite the lack of information in the system, it instantly arranges (many participants in the information environment begins to discuss a topic unexpectedly) (Fig. 1, a). It is clear that the sources of low entropy are the information here from the outside or being under SMM impact. An apologist of the Next Generation of "Media 2.0 concept" is obliged to object that in this case, the source of external information does not necessarily have to be traditional media that themselves are excited by the same pattern, responding to some events (the same nomination to candidates Sarah Palin in former 2008 US Vice-President's Elections or Volodymyr Zelenskiy nomination in recent 2019 Ukrainian President's Elections). 
Social media - Twitter, Facebook, and various blogs - are built on the similar principle, as opposed to traditional media with their hierarchical editorial policy [5].

This is a peer-to-peer, non-hierarchical info communication network with peer-to-peer agent nodes (individual users' interaction of the P2P type), as shown in [6]. The processes of ordering/excitement are one-sided, so it is safe to assert with a high degree of certainty that exogenous events (most often found to be frequent for the blogosphere) are induced by traditional media just like long-lived trends, for example, in Twitter. Otherwise, it will have to divide the blogosphere into "true new media" and "untrue", which already contradicts the non-hierarchical ideology concept of "Media 2.0".

The problem statement. That is, knowing basic principles of these two evolutional processes it is possible to try to impact the informational, or, more correctly, cyber space with inducing and/or controlling desirable informational events as a part of state or commercialized SMM strategy. And, introducing, configuring, adoption of info communication platforms to deploy appropriate technologies of such impacts' automation is not known to authors from open literature sources of very last years. This work is intended to describe possible scenarios of informational impacts in cyberspace and draw the ways of their efficiency increasement. On our opinion, in the modern world this kind of informational impacts is very important to save the leadership of the state, commercial presence, cyber supremacy using modern ICT environment appliance.

The structure of the paper. Chapter 2 of this paper is dedicated to the brief theoretical description of possible scenarios of informational impacts propagation in cyberspace using Omori tectonic theory. Subchapter 2.1 describes pure endogenous information event with controlled post-impact; subchapter 2.2 is dedicated to the support of quasi-endogenous information events with hidden pre-impact and controlled post-impact; and, finally, subchapter 2.3 briefly describes quasi-exogenous information event with no pre-impact and controlled post-impact case. After that in the chapter 3 let's make generalization of algorithmic basics for the implementation of the strategy of controlled social impacts in the information space. A visual algorithmic description of SMM kind information operations associated with both the target distribution of a given information content and the collection of data on response changes in the preferences and sentiments of various user groups has been given. Subchapter 3.1 is dedicated to the statement of the classification problem for audience profiling procedures into ICT environment that supports informational space to reach the targeted groups of electronic services consumers effectively. The hierarchical classification of the target audience profiling levels for ICT content distribution platforms that sharing key messages in e-government platforms over the information space has been contributed. And subchapter 3.2 describes the authors opinion on features of SMM technique implementation applicable to the strategy of managed social impacts based on e-government ICT platforms. Chapter 4 is devoted to the brief analytical case study of audience response dependence on some events, including controlled impacts and declared approaches feasibility preliminary assessment. Subchapter 4.1 shows the qualitative comparison of presented in the paper dependences with statistical processes, available from existent literature sources. And subchapter 4.2 presents the empiric study results discussion of live audience response dependencies on controlled impacts and their efficiency. The discussion and conclusions are also presented in the chapters 5 and 6 respectively.

\section{Some theoretical basics of the Omori tectonic theory and description of the options for sce- narios of informational impacts propagation in cyberspace}

Let's make a hypothesis that, evidently, it is possible to create a public resonance over short period of time, with the given amplitude, both with the help of the right endogenous precursors (key elements of the information presence or SMM strategy), and to manage it using a sequence of influences, while fixing how they affect in a feedback system on the basis of which allows to describe the moments of the compensating influences that lead the whole segment of system to a state of quasi-constant resonance (both for the endogenous and for the exogenous reaction of the target groups of society). Let's describe these processes on the basics of Omori tectonic theory for earthquakes shocks intensity description applicable to the targeted audience response. 
Works $[4,7]$ give the description of Omori's law, as:

$$
n(t)=\frac{K}{(t+c)^{p}}
$$

where $t$ is time; $K, c$ and $p$ are constants, and cumulative number of aftershocks after the main stress (or informational event peak size) could be defined by integral:

$$
N(t)=\int_{0}^{t} n(s) \mathrm{d} s=\frac{K\left[c^{(1-p)}-(c+t)^{(1-p)}\right]}{p-1} .
$$

By [8] and equations (3), (4) from [7] the seismicity rate as a function of time after the stress step is expressed as:

$$
R(t)=\frac{r \dot{\tau} / \dot{\tau}_{r}}{\left[\frac{\dot{\tau}}{\dot{\tau}_{r}} e^{\left(-\frac{t_{r}}{t_{a}}\right)}-1\right] e^{\left(-\frac{t}{t_{a}}\right)}+1}
$$

where, conditionally, $t_{a}$ is the characteristic relaxation time; $t_{r}$ is peak impact recurrence time; $r$ is the reference seismicity rate, proportional to the reached peak-event intensity level (the maximal frequency of key words occurrence) into informational space (Fig. 1).

By [1] the endogenous events' cumulative distribution of event-size $E$ is similar to the Gutenberg-Richter law with a parameter $\beta$ of 0.574 , that is typical for earthquakes (attenuation of social influences and inflammation of these effects by after and foreshocks), and for an exogenous case it could be fitted by this law with exponent 1.003 (when a contribution of introduced external impacts prevails).

Gutenberg-Richter law, where events' intensity $E$ is proportional to radiated seismic energy (discussion intensity propagation of an event within some targeted group):

$$
P_{r}\left(E^{*}>E\right) \propto E^{-(\beta+1)} .
$$

Let's also give the Omori law interpretation within view of (4) for intensities of fore- and aftershocks while informational events evolve within certain audience inside a cyberspace. For exogenous events and impacts equation (6) exists only:

$$
\begin{aligned}
& w_{i}\left(t<t_{0}\right) \propto\left(t_{0}-t\right)^{-\alpha_{g}}, \\
& w_{i}\left(t>t_{0}\right) \propto\left(t-t_{0}\right)^{-\alpha_{d}} .
\end{aligned}
$$

Here $w_{i}(t)$ is the frequency (occurrence) of $i$-th key words at the time $t$ (or nearest time interval). Let's follow with considering scenarios of informational impacts in the cyberspace while maintaining SMM automation by ICT means.

\section{1. Scenario 1. Pure endogenous information event with controlled post-impact re-} sponse

With this scenario, first it is necessary to detect new trend in the society or targeted social group, and to define right key words to impact this group, late to decide about expediency of controlled impact support after event peak size time moment $(t=0$, Fig. $1, \boldsymbol{b})$. This could be performed by monitoring algorithm presented in Fig. 2 (key messages monitoring in the informational space and probably by using certain kind of deep learning algorithms, neural networks etc.). This algorithm could be realized by means of implementing the scalable metadata fixation 
platform, applicable approaches are described in the works [9-11]. An additional keywords injection process is depicted in Fig. 3. If controlled endogenous aftershock impact is desirable, it is possible to implement (voidable) algorithms in Fig. 4, 8 (key messages injection process to support controlled social impact and to stabilize the impact intensity during $t_{a}$ aftershock period), where $f_{\text {ms.inj. }}$ is proposed, as equivalent of corrective (compensation) key messages injection intensity (at the time $t$ ) within aftershock active impact period (up to $t_{a}$ time) and could be assessed in respect to (1)-(3) as:

$$
f_{m s g . i n j .}=w_{i}\left(t_{0}\right)\left(1-\frac{w_{i}(t) R(t)}{w_{i}\left(t_{0}\right) R\left(t_{0}\right)}\right),
$$

and event-size for key words set $i$ at peak-time $t_{0}\left(t_{0}=0\right)$ is taken by [1]:

$$
E_{i, t_{0}}=\frac{1}{2 T+1} \frac{w_{i}\left(t_{0}\right)}{\sum_{t=t_{0}-T}^{t_{0}+T} w_{i}(t)},
$$

where $w_{i}(t)$ is the frequency (occurrence) of key word $i$ at the time $t$. For our informational impact's propagation, it is possible to put a relaxation time $t_{a} \approx T($ Fig. 1, b).

In the case of possible negative compensation intensity appearance, it means, that it is necessary to apply lawful interception and filtering measures for key messages on the state level or try to introduce new impact to the targeted groups and snuff out current superfluous resonance in the cyber space. In any case, the duration and expediency of influence is determined by the volume of allocated resources, as well as by the criterion of accomplishing the goals set of SMM strategy.

Let's note that it is necessary to choose $t_{a}>t_{r}$ to extinguish or change the subject of impact in a managed system with social influence and $t_{r}>t_{a}$ for long-term maintenance of the level of managed social impact with injection of key messages considering (7), (8); where $t_{r}$, as recurrence time could be calculated by solved equation (3) (where $\tau_{r}$ and $\tau$ are the stressing rate prior to and following the stress step, and $r$ is proportional to $E_{i, t 0}$, and taking $t_{a} \approx T$ ):

$$
t_{r}=T \ln \left(\frac{\frac{\dot{\tau}}{\dot{\tau}_{r}} \cdot R(t)}{R(t)\left[1-e^{\frac{t}{T}}\right]+r \frac{\dot{\tau}}{\dot{\tau}_{r}} e^{\frac{t}{T}}}\right)
$$

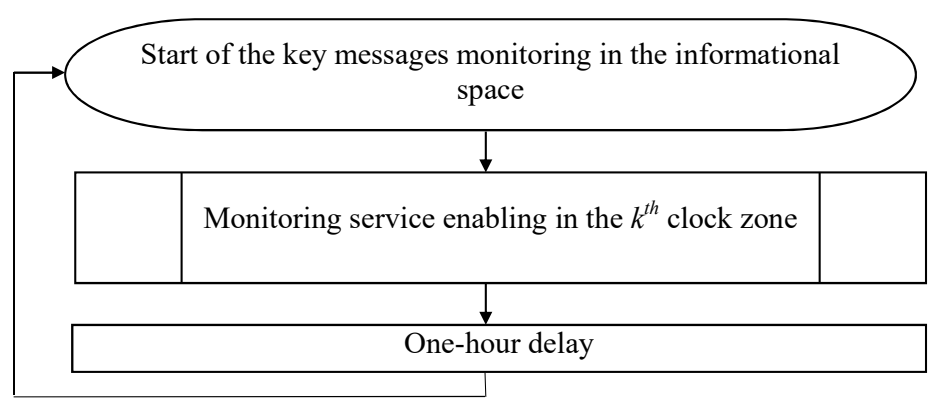

Fig. 2. The basic algorithm of key messages monitoring in the informational space

The difference between Fig. 2, 3 is that first one is intended for passive monitoring of informational space or digital media resources, and the second one is intended to realize controlled (hidden) pre-impact, to reach, respectively, defined event peak intensity in some SMM strategy activities' profile. Time-zones are also featured in the case if scalable strategies are realizing. 


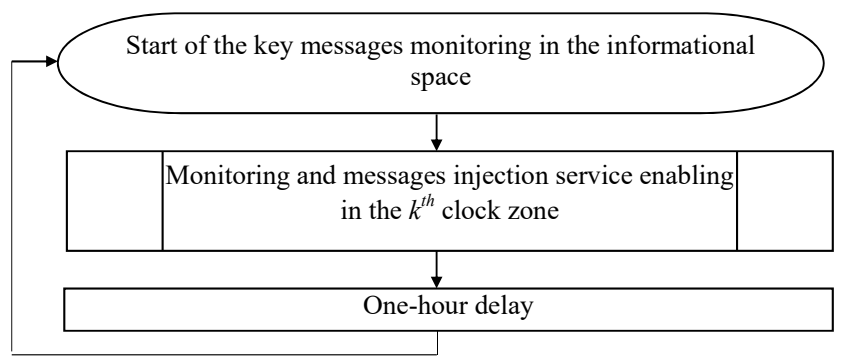

Fig. 3. The basic algorithm of monitoring and injection of the key messages to informational space

\section{2. Scenario 2. Quasi-endogenous information event with hidden pre-impact and con-} trolled post-impact response

Within this scenario, it is firstly necessary to determine hidden pre-impact beacons for preparation the society (or audience) to react on some informational event very intensively (Fig. 1, b). Calculations of the necessary time profile for intensities of hidden preliminary pre-impacts (sub active phase) should be performed using (7) and (8) and considering desirable event-size $E_{i, t 0}$, $T$ - the period for preparation, and, accordingly to the used algorithm of monitoring and injection of the key messages to informational space, involving ICT mechanisms (Fig. 3). If controlled aftershock impact is desirable, it is possible to implement algorithms in Fig. 3, $\mathbf{5}$ applied mandatory by Fig. 7, using Fig. 4 after reaching of pre-defined event peak size limit $E_{i, t 0}$ into the cyber (informational) space to stabilize the impact intensity during $T$ aftershock period.

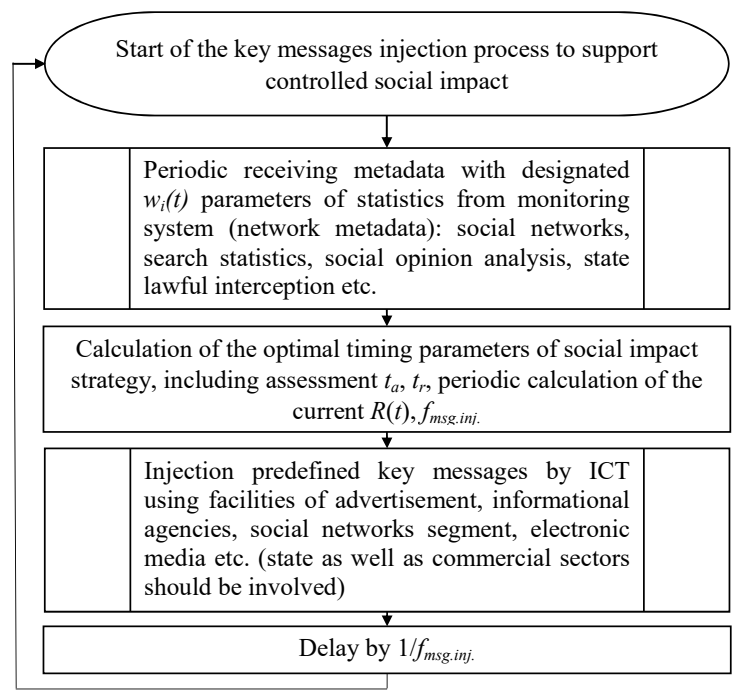

Fig. 4. The algorithm of the key messages injection process to support controlled social impacts

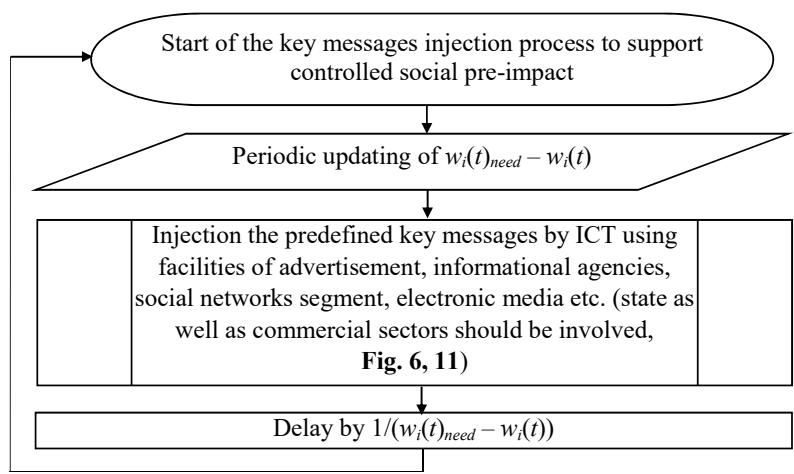

Fig. 5. The algorithm of the key messages injection process to support controlled social pre-impact 
Fig. 6 presents a means set of ICT platforms and their deployment process to support informational impacts in the targeted cyber space segments. Thus, for endogenous influences scenarios it is necessary to evaluate amplitude of influence and make selection of $t_{a}$, $t_{r}$ in order to maximize (to reach desirable amount of) $E_{i, t 0}$ and $R(t)$ in real time based on the results of feedback analysis of statistical indicators, in particular statistics for dedicated keywords $w_{i}(t)$, for example by feedback from the infocommunication environment by metadata fixation system, Fig. 6.

The designing features of cloud messaging protocol for content distribution as a service is studied in [12], and respective architectural concepts are presented in [13], and also in [14]. Let's also note that all social networks and media distribution platforms use cloud engines to operate with higher resource efficiency.

It is important to determine correctly targets and targeted geographical regions/audience for social impact informational injection by ICT and intensity timing profile of social informational impact (chosen SMM strategy) that supported by ICT to calculate the features and scalability potential of such technical platforms in the future works. That is, the further researches on ICT distributed platforms adaptation (feedback collection features, maximal load in specialized architecture for impact propagation assessments) should be performed in the future. Mentioned architectural features should consist of e-services plurality, and servers configuring, impact propagation in networks flowcharts, results of proposed techniques implementing and automation.

\section{3. Scenario 3. Quasi-exogenous information event with no pre-impact and controlled} post-impact response

For this case scenario implementing $($ Fig. 1, a) it is necessary to define initial key event to determine the most effective moment for implementing the exogenous impact using Fig. 2, and mandatory by Fig. 8, 4 algorithms. Let's note that this is very simplified approach and do not consider deep learning algorithms formalization procedures here, as it could be the subject of special and stand-alone investigation. The algorithm in Fig. 4 is used after reaching of pre-defined event peak size limit $E_{\text {limit }}$ into the cyber (informational) space to stabilize and control the impact intensity during $t_{a}$ exogenous aftershock period. The rest features of implementation were described in the first scenario. All these presented algorithms consider separate impacts within pre-defined clock zones during ongoing SMM strategy realization. Let's note, the respective effects to the targeted audience could be got using commercial messaging services (Fig. 6), for example by Viber commercial solutions, Google Adds, Facebook, Telegram services etc.

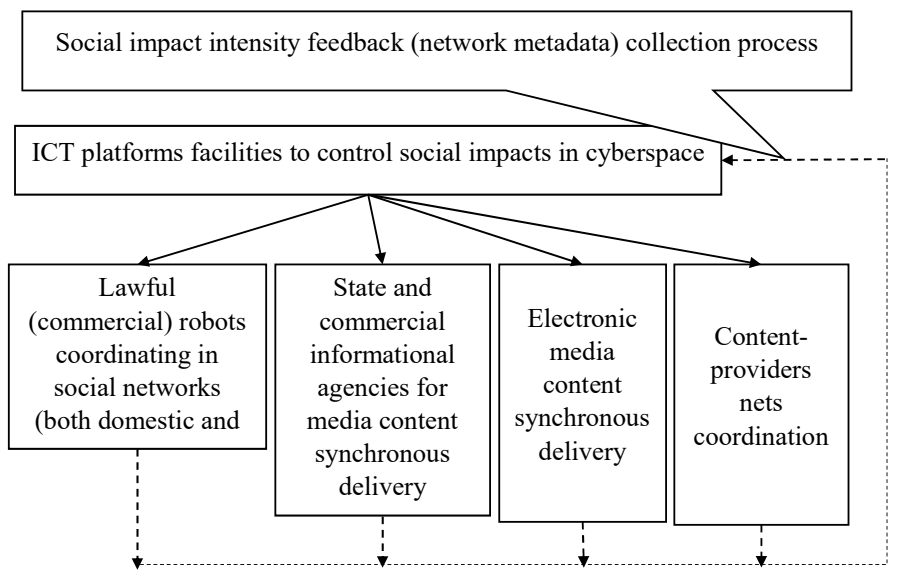

Fig. 6. A means of ICT platforms and their deployment process to support informational impacts in cyber space during ongoing SMM strategy realization

Fig. 7 depicts the algorithm of key messages monitoring service enabling in the $k^{\text {th }}$ clock zone to be realized within proposed scenario described in subchapter 2.2. 


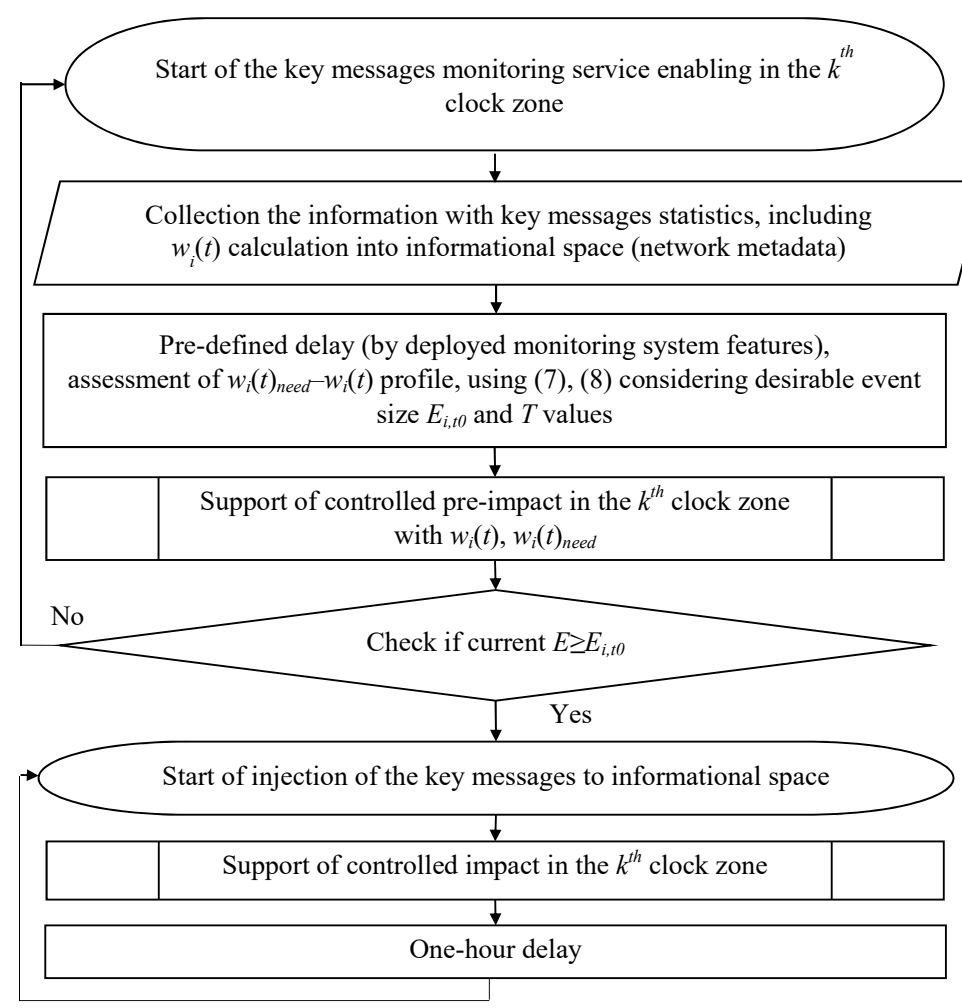

Fig. 7. The algorithm of key messages monitoring service enabling in the $k^{\text {th }}$ clock zone

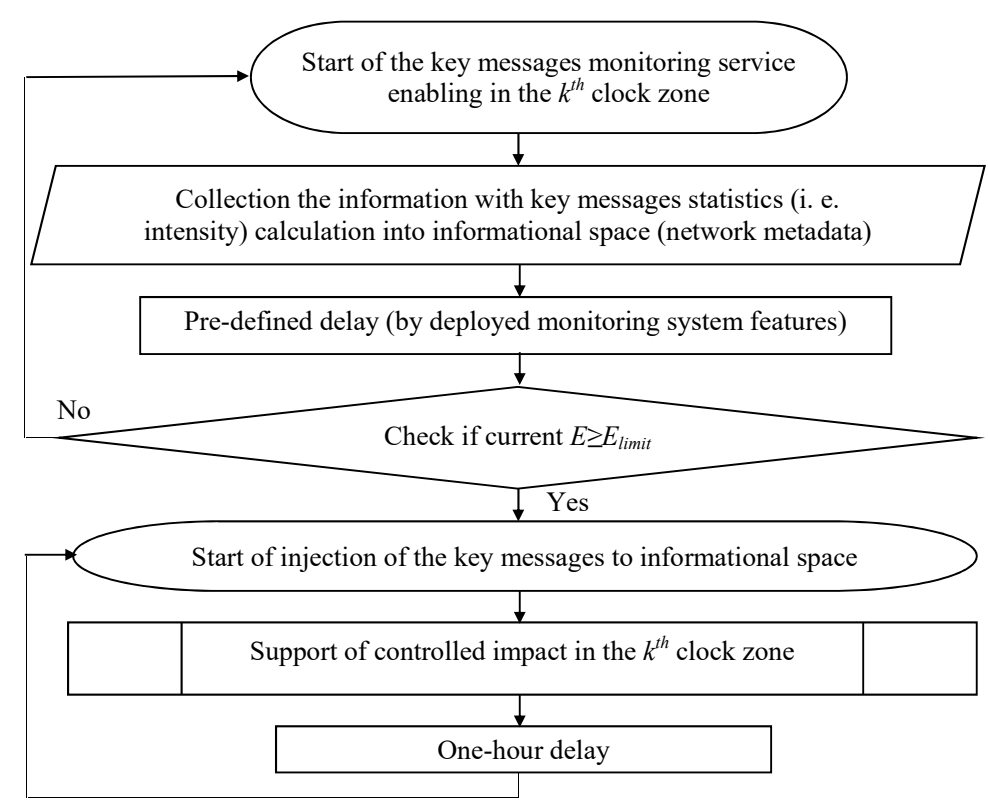

Fig. 8. The algorithm of the key messages monitoring service enabling in the $k^{\text {th }}$ clock zone for exogenous impact preparation strategy

In Fig. 7, 8 module "Support of controlled impact in the $k^{\text {th }}$ clock zone" could be realized using facilities that have been described and depicted in the following section 3 of this paper.

3. The generalized approach to the implementation of the strategy of controlled social impacts in the information space

To date, the data on user activity in the world information space has become a valuable information resource. Hidden, but credible reports (from casual life practice) about leading glob- 
al IT companies such as Google, Facebook, Twitter, Microsoft, MasterCard, VISA, booking and reservation services and many others involved, probably make possible to postulate that them use their products to provide B2B collaboration between different companies in order to get the most complete profile of the persons who consuming their or third-party digital services. The purpose of obtaining such information is not only the selection of a content for its personalized preferences and sentiments [15, 16], for SMM introducing but also realization of unprecedented cases of information operations related to both targeted advertising [5, 17], as products, goods and services, and political forces, social programs of the state, as well as with the manipulation of the opinion of representatives of certain social groups, that is, the creation of targeted social influences, in some special cases operations of cyber-fight on the territory of the enemy, etc. Information and telecommunication platforms, with the help and on the basis of which the above-mentioned activities are realizing, as a rule, are simultaneously platforms of state e-governance or incorporated. Support for information operations and SMM automation on their new ones becomes most effective in the case of targeted segments of an audience, which profiled and integrated into target groups based on the characteristics that are posed when implementing a strategy of managed social impacts (Fig. 9).

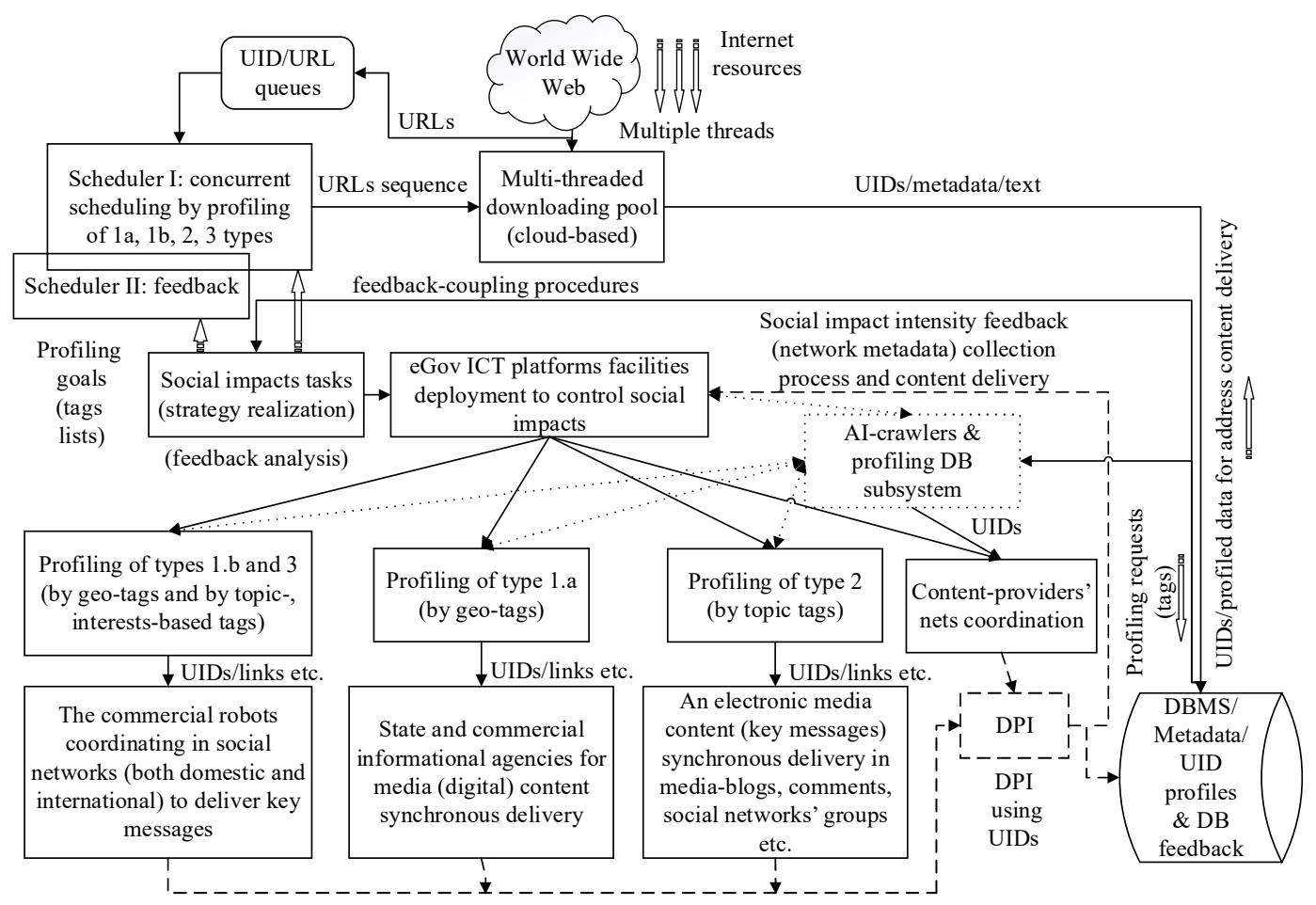

Fig. 9. The generalized approach for implementing the strategy of controlled social impacts into the information space

In Fig. 9 the DBMS/Metadata/UIDs profiles module should perform the UIDs collection and storing process (under respective State DB Administration advising); and late binding the collected metadata to collected UIDs, which data space is organized under UID's unification policy (by format) for e-mail addresses, web-addresses, links to additional content, users' pages links to social networks/forums etc. This forming DPI and web-crawling results that could be used furtherly in feedback analysis process.

\section{1. The statement of the problem of SMM automation into ICT environment}

According to [18-20], typical examples of information impact generators are:

- Social networking groups;

- Small forums with the community of their participants; 
- Sections or topics of large forums with a community of forum members who are active in a section or topic;

- Thematic interactive sites or blogs and the commentators' communities;

- Thematic sections of the online media and the commentators' communities.

Thus, profiling levels of the target audience for infocommunication complexes for delivering key messages in e-government platforms in the process of managing social impacts (or while realizing SMM strategy) could be classified and presented as following (ordered by decreasing the performance):

1a. Managed broadcasting of key media messages (in mass media), broadcasting targeted advertising messages and content in the media, for example, social advertising, for which the necessary coverage area should be determined, or certain geographic areas.

1b. Managed broadcast of commercial advertising and content or social content in social networks, for which it is necessary to determine the target audience based on the territorial principle, using the meta-information collection (geo-tagging) in the database (DB) of the electronic communication platform of the e-government Artificial Intelligence (AI) subsystems using web crawlers (web-spiders) - programs that are part of search engines. And, respectively, Internet-driven web pages analyzing to enter information about them (key-words) to a database (Fig. 10) [21]. According to the definition of Wikipedia, spider programs perform a general search of information on the Internet. They report the contents of the found document, index it and extract the summary information. They also read headers, some links, and submit the indexed information to the search engine database. The prototype of powerful crawler architecture that could be implemented in the cloud environment is presented, for example in [22].

2. Group distribution within the framework of implementing the strategy of managed social impacts of key informational messages (target media content) to members of social networking groups and forums, their sections, commentator communities of thematic interactive sites, including sections of electronic mass media. To determine and create a target audience database, it is necessary to use artificial intelligence subsystems (AIs) using web crawlers that search for relevant topics, key words, etc.

3. Individual targeted mailing of key messages to profile users of social networks and other ICT systems whose UIDs lists are determined by a set of keywords (subject topics, user interests) that are captured by the web crawler AI subsystem for the implementation of a certain SMM strategy for managed social impacts.

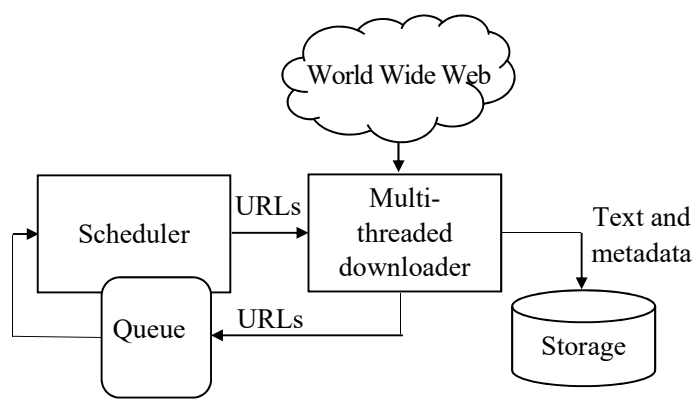

Fig. 10. The basic architecture of the typical Web-crawler software tool (by Wikipedia), by $[21,22]$

For the implementation of all three proposed levels of profiling of target audiences for infocommunication delivery systems of key messages in e-government platforms, it is possible to use metainformation fixation systems with the use of DPI (Deep Packet Inspection) technologies (Fig. 11). This architectural direction in relation with inter-cloud messaging protocol development for content distribution as a service (of CoDaaS type) over Future Internet is for example investigated and discussed in [12]. Algorithms and generalized strategies for the implementation of computer-aided SMM influences are developed by the authors of this publication and could easily be automated by IT companies. 


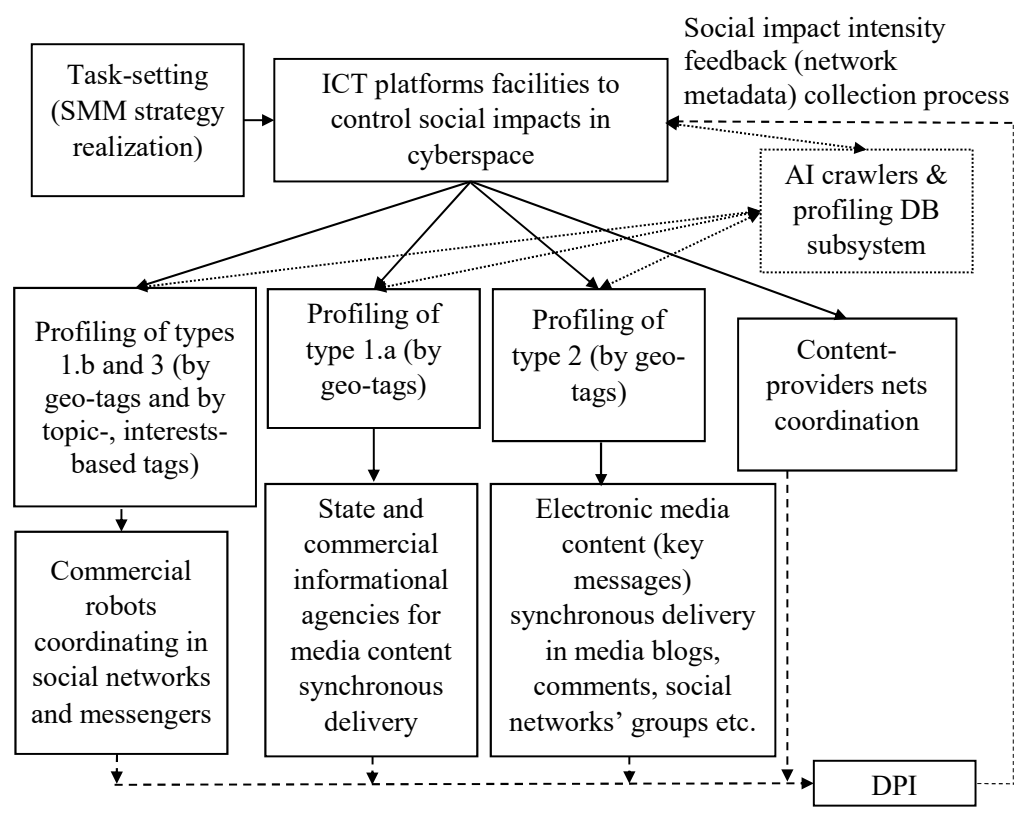

Fig. 11. Means of eGov (ICT systems) platforms and their deployment to support information impacts into social and communication space, with proper targeting the audience

(performed by the authors)

The algorithms that improving the storage efficiency of DPI technological applications and respective database management principles are discussed in [32], and semantic principles of such analysis are also given and developed in the works $[18,20]$.

\section{2. The features of implementation of the strategy of managed social impacts based on E-Government ICT platforms}

An important aspect of the functioning of e-government platforms for managing information impacts is the monitoring of metadata in the Internet space [1] (particularly, in the socio-infocommunication environment) (Fig. 9, 11). Content providers operating within the existing national legal field may be required to configure their networks in such a way as to elaborate the concepts of the introduction of e-government systems and provide secured data-based services in accordance with the processes of the operation of the established infocommunication platform (Fig. 10). Feedback-coupling mechanisms are also needed to control the effect of the implementation of certain information operations and the possible automated adjustment of the implementation of the strategy of e-government or advertising campaigns of SMM kind on selected groups of users of certain information resources. An important feature of searching through search engines is the need to create a single informational field with unique identifiers of these or other user profiles (global UIDs). But this, in turn, requires the creation of a reliable information storage and information security measures that should be developed at the state level on the hybrid ICT platform (Fig. 9, 11). Relevant robotic crawling devices should have a special mode of access to Internet-based traffic points due to the specifics of their operation and the large number of consistent requests [22]. Distribution of commercial or other type of information in such socio-communication environments as social networks is rather complicated process [2, 3], therefore its course should be managed using means of artificial intelligence. Accordingly, the development of such tools is one of the priorities for the creation of e-governance systems for the future. Their application will facilitate the coordination of efforts of various organizations of commercial and public character within the framework of established systems for controlled social impacts and their protection in the national information space as automated SMM strategy realization, including from potentially hostile multinational corporations. 


\section{A brief analytical case study of audience response dependencies on controlled and casual impacts}

Authors should note that analytical (statistical) proof of the dependencies (Fig. 1), presented in this work faces with difficulties due to different reasons. And, to some extent, these difficulties highlight the partially debating character of the paper, which scope was to present idea and draw narrative ICT approach of the controlled impacts realization (of SMM kind) under specified targeting audience, being used as a part of state informational policy implementation within respective e-Gov services deployment. The main difficulty (Fig. 9) is using the specialized technique, named DPI or lawful interception. It means that effective realization of such ICT platforms, regardless of their commercial or state ownership, faces with specified law constraints, being a kind of data intelligence process [23] and obtained data, regardless of its volume are as usual classified (as by the US regulations, as well as in EU, PRC, and another countries). It means also that data sets, collected using such approaches are in the property of state or commercial structures and cannot be published without their permission, even for scientific purposes. But authors could strongly agree with readers that this situation does not satisfactory, and providing in this paper result of their own alternative study.

\section{1. The comparison with statistical processes, known from existent literature sources}

First, let's compare character of dependencies in Fig. 1 [1] with results of Twitter crawling that were made in [24] and [25]. These works represent the information propagation (twits' volumes) about Abu Dhabi double-crime event (2014) and Sebastian Vettel victory in Formula 1 (2013), respectively. The correlation of time synchronized curves from [25] and of Fig. 1, $\boldsymbol{a}$ gives the correlation index 0.3802 and respective index 0.4416 to Fig. 1, b. For this case, the intensity growth of audience response was smooth. It means Fig. 1, $\boldsymbol{b}$, that discussion was growth inside the society (is more endogenous), being ad initium quasi exogenous. Therefore, it is suddenly that the event of Sebastian's Vettel victory in F1 competitions (2013), studied in [24] is much more endogenous (correlation indexes are 0.62 versus 0.1 , respectively), as the attributes of hidden advertisement and publicity hooks are, evidently, were presented during that campaign. It could be concluded from this that used material by [1] in general is correct and could be used for further scientific investigation process.

\section{2. The empiric study of live audience response dependence on controlled impacts and its efficiency evaluation}

Secondly, let's analyze 50 data sets, generated from certain video files which contain recordings of Ukrainian TV talk-shows with the echo of the indexes of positive/negative reaction of the auditorium is made, according to the topic of the discussion. It is postulated that a sample of more than 300 viewers present in the television studio is representative of all sections of society to a sufficient extent (justified by TV-channel sociologists), and their moods and preferences are aggregated and become an anonymous echo of the audience in the running echo graph (using also remote interactive ICT means), and the audience fairly adequately represents certain target groups that would be formed in the Internet space by topic and preferences, in relation to the topics covered in a particular television program. Let's detect the typical time profile of the audience response during all the program [26] (the properties of analyzed materials are substantially equivalent to the retrieved from mentioned media), Fig. 12 (accomplished by authors, empirical data, no simulation was used).

Authors specially do not show the process of data retrieving, as this is out of scope for this paper's topic but interested readers could contact authors personally to get some data set in the table format. Let's note that this process could be automated using Microsoft Azure toolset and appropriate software deployment, to detect necessary keywords. As for this case the keywords used were the following (in Ukrainian): "president, system, responsibility, referendum, reform". The endogeneity of the audience's reaction prevails (the correlation index to the Fig. $\mathbf{1 ,} \boldsymbol{b}$ is about 0.30 versus 0.18 for this index to Fig. 1, a). The keywords usage occurrence profile is quasi-exog- 
enous (the correlation index to the Fig. 1, $\boldsymbol{a}$ is about -0.03 versus -0.23 for this one to Fig. 1, b). The efficiency of the impact's propagation inside the audience by row correlation index of keyword occurrence and audience response intensity is 0.17 . For the reaction intensity the $t_{r}$ index value is about $40-48$ minutes, and $t_{r}>t_{a}, t_{a} \approx 5-6$ minutes.

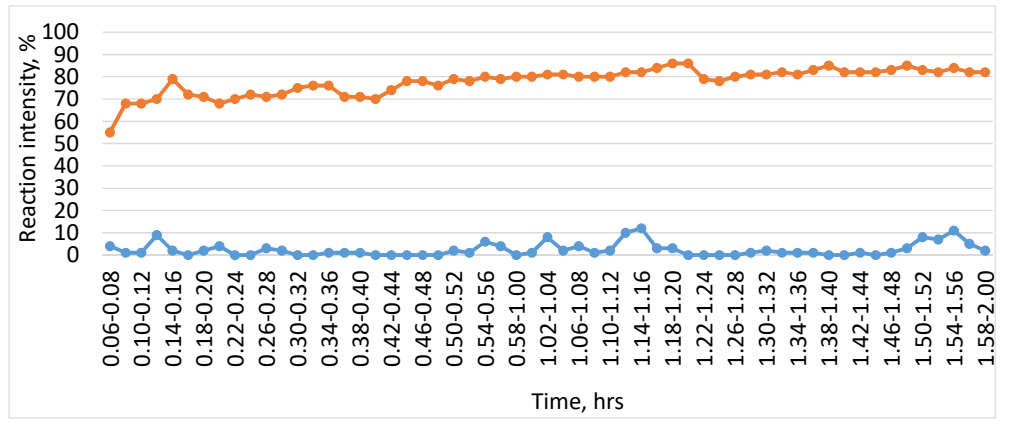

Fig. 12. The temporal profile of audience response intensity against keywords' usage occurrence (the empiric data are gathered)

A data from Fig. 12 could show that the medium reaction level is $80 \%$. This case is studied with condition of controlled impacts absence.

The next case is more interesting, as one from the authors of this paper could be represented in the editorial office during the TV program recording (the properties of analyzed materials are substantially equivalent to the retrieved from recorded media [27]) and conditionally could made hidden advices to the linkman how often to control the discussion, using certain calculations, based on the pre-collected dataset. So, hypothesis, postulated in this paper were put under verification by respective series of experiments.

The respective temporal profile of audience response intensity against keywords' usage occurrence is shown in Fig. 13 (accomplished by authors, empirical data, no simulation was used).

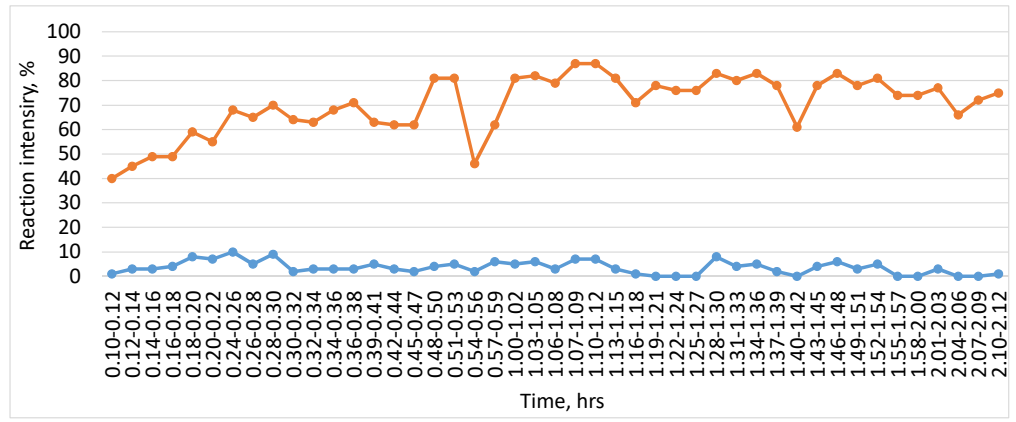

Fig. 13. The temporal profile of audience response intensity against keywords' usage occurrence in the case of controlled impact realization (the empiric data are gathered)

This TV program recordings were analyzed and for this case the keywords used were the following (in Ukrainian): "demarcation (peace), peace, negotiations, conflict, invasion, Minsk treaties, fight (protect), corruption". Analyzing Fig. 13 let's note that there are signs of exogeneity of the audience's reaction (which is cardinally different to previous case and typical for hidden impacts in more than $80 \%$ of such cases) at the level of informative (and partly emotional) attitudes from the participants of the discussion (the correlation index to the Fig. 1, $\boldsymbol{a}$ is about -0.06 , but this index to the Fig. 1, $\boldsymbol{b}$ is less than -0.03 , so this is ambivalent situation, or hidden exogeneous reaction). The keywords usage occurrence profile is endogenous (the correlation index to the Fig. 1, $\boldsymbol{b}$ is about 0.21 versus -0.18 for this one to Fig. $\mathbf{1}, \boldsymbol{a}$ ). The efficiency of the impact's propagation inside the audience by row correlation index of keyword occurrence and audience response intensity is 0.31 (that is up to $45 \%$ (!) greater, than for previous case, Fig. 12). And the times for impacts ac- 
tivization were assessed by (9), $t_{r}$ index value is chosen and implemented (retrieved from Fig. 13) as about 12-14 minutes, and $t_{a} \geq t_{r}, t_{a} \approx 16-20$ minutes. Thus, scenario 2 was implemented (using appropriate time scaling).

A data from Fig. 13 could show that the medium reaction level is $74 \%$, so the energy of audience response is more constant, being "smeared" over time, accordingly to (4). The controlled reaction overcomes the negative expressions about the subject of discussion, making the disputing more constructive.

\section{Discussion of the development features of the Basic Concept for ICT Platforms Deployment Strategy for Social Media Marketing}

As for disclaimer, this work could be partially considered as positional authors' look. It was not performed as a part of project, supported by any organization/government. All investigation should be considered as the private initiatives of authors. But all described processes of informational impacts are considering to be realized and automated into the models of specialized ICT infrastructure for further investigations. Being highly significant for the developing of the partnership between business, government and citizens, a part of this work could be transformed on the commercialization basis, especially in SMM automation field.

Let's note that high informational impact intensity stabilizing during $t_{a}$ aftershock period by means of ICT (Fig. 6) is a sort of cyber operation and should be performed carefully under control of respective state services and in the interests of the state due to its potentially destructive character [28-30]. Especially, when significant targeted resonance is created within short period of time. It is very important to define goals, subject and components of informational cyber impact correctly, as well as targeted geographical region and audience coverage, including traditional media, and by means of state or commercial advertisement agencies' resources (as for example), depending on amount of allocated resources. Ways of ICT means effective application, including cloud resources virtualization and scaling is also very important question in the national scale systems [31] to be investigated in the future.

Interesting is also approach with creation of countermeasures to the undesirable hidden informational impacts while they are not appeared in the form of highly resonance informational peak-event, by setting some "sematic jamming" with specially adopted contrary key words or lawful interception (of DPI kind) traffic filtering. In the active phase, while resonance into informational space is undesirable and is high it should be considered inspiring a new exogenous impact using inequality $t_{a}>t_{r}$ and assessments by (9) to extinguish or change the subject of current impact (or to struggle current strategic trend) in a managed system with targeted social influence in some region or social group. But this technique could be the subject of further study.

As was already noticed, correct determining of targets and targeted geographical regions/ audience for social impacts informational injection by ICT and intensity assessment of such impacts by ICT to calculate the features and necessary scalability potential of the respective infocommunication architectures are mutually significant tasks. Nearest investigations will be also addressed to the technical side of the proposed approaches realization with support of their automation by means of ICT.

The necessity of attention of the state to the issues raised in this work is undoubted, since the benefits from active interaction with society consists not only in carrying out of certain information campaigns, but its consequence, in essence, is the achievement of the top of the process of society informatization in the postindustrial era, which will directly contribute to the consolidation of a single monolithic nations.

\section{Conclusions}

Therefore, in this paper, the general statement of the problem of implementing the strategy of controlled social impacts in the information space based on hybrid intelligence ICT platforms is considered. Thus, as a result of investigations conducted within this work, it is possible to conclude the following. 
A generalized visual algorithmic description of information operations (of SMM sort) related to both the targeted distribution of the given information content and the collection of feedback data on reactionary changes in the preferences and sentiments of certain groups of users in the relevant social and informational environments is presented (three scenarios were depicted).

The classification of types of users' profiling of digital services in the information space has been contributed (three types were described), which can be practically useful in the development of e-government systems on the basis of modern ICT tools. A brief characteristic of the profiling process to arrange a target audience for the purpose of carrying out the above-mentioned information operations on the basis of infocommunication platforms operating in an open Internet space is given. Authors find an effective the transition to hybrid cloud information and communication technologies for the implementation of the processes shown in Fig. 9.

As could be concluded from Fig. 12, 13 (using empirically collected data) the approaches that presented in this article could significantly improve the efficiency of the impact's propagation inside the targeted audience using controlled social impacts (SMM-based) technique. It is possible to contribute, as a result of empiric experimental data sets analysis, an efficiency evaluation index of the impact's propagation inside the audience by row correlation calculating of keyword occurrence and audience response intensity (in the conditions of pre-defined impacts absence this index is about 0.17 , otherwise it was increased up to 0.31 , or by $45 \%$ ).

Developed mathematical description helped to assess the timing parameters for live impact propagation control purposes. It has been empirically shown that it is necessary to choose $t_{a}>t_{r}$ to extinguish or change the subject of impact in a managed system with social influence and $t_{r}>t_{a}$ for long-term maintenance of the level of managed social impact with injection of key messages (from Fig. 12: $t_{r}=40-48 \mathrm{~min}, t_{a}=5-6 \mathrm{mins}$; and from Fig. 13: $t_{r}=12-14 \mathrm{~min}$, $\left.t_{a}=16-20 \mathrm{mins}\right)$.

The authors are planning to conduct a further and deeper analytical empiric study to confirm in more accurate manner the postulated in this paper target audience reaction intensity dependencies on the keyword occurrences which used to impact on this reaction character. For this purpose, more issues of information-analytical television programs will be taken, during which the echo of the indexes of positive / negative reaction of the auditorium is made, according to the topic of the discussion. Social networks data dynamics study is also planned to be used.

\section{References}

[1] Klimek, P., Bayer, W., Thurner, S. (2011). The blogosphere as an excitable social medium: Richter's and Omori's Law in media coverage. Physica A: Statistical Mechanics and Its Applications, 390 (21-22), 3870-3875. doi: https://doi.org/10.1016/j. physa.2011.05.033

[2] Shinyaeva, T. S., Tarasevich, Y. Y. (2016). Virtual network as excitable medium. Journal of Physics: Conference Series, 681, 012008. doi: https://doi.org/10.1088/1742-6596/681/1/012008

[3] Szell, M., Grauwin, S., Ratti, C. (2014). Contraction of Online Response to Major Events. PLoS ONE, 9 (2), e89052. doi: https:// doi.org/10.1371/journal.pone.0089052

[4] Utsu, T., Ogata, Y., S, R., Matsu'ura (1995). The Centenary of the Omori Formula for a Decay Law of Aftershock Activity. Journal of Physics of the Earth, 43 (1), 1-33. doi: https://oi.org/10.4294/jpe1952.43.1

[5] Yoo, S.-C., Shin, I. (2018). Digital Signage Media Creative : A Study for the Media Typology and Advertising Creative Strategy for Digital Signage. The Korean Journal of Advertising, 29 (6), 81-108. doi: https://doi.org/10.14377/kja.2018.8.31.81

[6] Dunbar, R. I. M., Arnaboldi, V., Conti, M., Passarella, A. (2015). The structure of online social networks mirrors those in the offline world. Social Networks, 43, 39-47. doi: https://doi.org/10.1016/j.socnet.2015.04.005

[7] Correig, A. M., Urquizú, M., Vila, J., Manrubia, S. C. (1997). Aftershock series of event February 18, 1996: An interpretation in terms of self-organized criticality. Journal of Geophysical Research: Solid Earth, 102 (B12), 27407-27420. doi: https:// doi.org/10.1029/97jb02487

[8] Dieterich, J. (1994). A constitutive law for rate of earthquake production and its application to earthquake clustering. Journal of Geophysical Research: Solid Earth, 99 (B2), 2601-2618. doi: https://doi.org/10.1029/93jb02581

[9] Poniszewska-Maranda, A., Matusiak, R., Kryvinska, N., Yasar, A.-U.-H. (2019). A real-time service system in the cloud. Journal of Ambient Intelligence and Humanized Computing. doi: https://doi.org/10.1007/s12652-019-01203-7 
[10] De la Prieta, F., Bajo, J., Rodríguez, S., Corchado, J. M. (2016). MAS-based self-adaptive architecture for controlling and monitoring Cloud platforms. Journal of Ambient Intelligence and Humanized Computing, 8 (2), 213-221. doi: https://doi.org/ 10.1007/s12652-016-0434-8

[11] Mladenow, A., Kryvinska, N., Strauss, C. (2012). Towards cloud-centric service environments. Journal of Service Science Research, 4 (2), 213-234. doi: https://doi.org/10.1007/s12927-012-0009-y

[12] Kaddouri, A., Guezouri, M., Mbarek, N. (2017). A new inter-cloud service-level guarantee protocol applied to space missions. International Journal of Grid and Utility Computing, 8 (2), 152. doi: https://doi.org/10.1504/ijguc.2017.085909

[13] Jiang, L., Feng, G., Qin, S. (2015). Content Distribution for 5G Systems Based on Distributed Cloud Service Network Architecture. KSII Transactions on Internet and Information Systems, 9 (11), 4268-4290. doi: https://doi.org/10.3837/tiis.2015.11.001

[14] Krishna, M. (2019). User-Centric and Information-Centric Networking and Services. CRC Press, 310. doi: https://doi.org/ 10.1201/9781315207650

[15] Molnár, E., Molnár, R., Kryvinska, N., Greguš, M. (2014). Web intelligence in practice. Journal of Service Science Research, 6 (1), 149-172. doi: https://doi.org/10.1007/s12927-014-0006-4

[16] Peköz, Ü. G. (2018). Product Usage Data Collection and Challenges of Data Anonymization. Lecture Notes on Data Engineering and Communications Technologies, 117-136. doi: https://doi.org/10.1007/978-3-319-94117-2_6

[17] Bauer, C., Kryvinska, N., Strauss, C. (2016). The Business with Digital Signage for Advertising. Lecture Notes in Information Systems and Organisation, 285-302. doi: https://doi.org/10.1007/978-3-319-28907-6_19

[18] Korzh, R., Peleshchyshyn, A. (2013). Formalization of process of forming university's information image in the social environments on the internet. Eastern-European Journal of Enterprise Technologies, 5 (3 (65)), 4-8.

[19] Korzh, R., Fedushko, S., Peleshchyshyn, A. (2015). Methods for forming an informational image of a higher education institution. Webology, 12 (2).

[20] Korzh, R., Peleshchyshyn, A. (2016). Forming University's Information Image Based on Image Information Generator. Journal of Multidisciplinary Engineering Science and Technology, 3 (1), 3621-3624.

[21] Witten, I., Frank, E., Hall, M., Pal, C. (2011). Data mining. Burlington, MA: Morgan Kaufmann.

[22] Lehtonen, J. (2011). Characterizing the deep Web. University of Turku.

[23] Cadwalladr, C., Graham-Harrison, E. (2019). Revealed: 50 million Facebook profiles harvested for Cambridge Analytica in major data breach. Available at: https://www.theguardian.com/news/2018/mar/17/cambridge-analytica-facebook-influence-us-election

[24] Alsaedi, N., Burnap, P. (2015). Arabic Event Detection in Social Media. Lecture Notes in Computer Science, $384-401$. doi: https://doi.org/10.1007/978-3-319-18111-0_29

[25] Burnap, P., Williams, M. L., Sloan, L., Rana, O., Housley, W., Edwards, A. et. al. (2014). Tweeting the terror: modelling the social media reaction to the Woolwich terrorist attack. Social Network Analysis and Mining, 4 (1). doi: https://oi.org/10.1007/ s13278-014-0206-4

[26] Pravo na vladu season 6 issue 39 online on $1+1$ video (2019). Available at: https://www.youtube.com/watch?v=AyfZ9EO9qEM

[27] Pravo na vladu season 6 issue 36 online on $1+1$ video (2019). Available at: https://1plus1.ua/1plus1video/pravo-na-vlast/6-sezon/ 36-vypusk-pravo-na-vladu-za-4-lipnya-2019-roku\#player

[28] Narayanan, A., Shmatikov, V. (2008). Robust De-anonymization of Large Sparse Datasets. 2008 IEEE Symposium on Security and Privacy (sp 2008). doi: https://doi.org/10.1109/sp.2008.33

[29] Salas, J., Domingo-Ferrer, J. (2018). Some Basics on Privacy Techniques, Anonymization and their Big Data Challenges. Mathematics in Computer Science, 12 (3), 263-274. doi: https://doi.org/10.1007/s11786-018-0344-6

[30] Weber, R. H. (2010). Internet of Things - New security and privacy challenges. Computer Law \& Security Review, 26 (1), 23-30. doi: https://doi.org/10.1016/j.clsr.2009.11.008

[31] Klymash, M., Demydov, I., Beshley, M., Shpur, O. (2016). Features of the cloud services implementation in the national network segment of Ukraine. Information and Telecommunication Sciences, 1, 31-38. doi: https://doi.org/10.20535/24112976.12016.31-38

[32] Yu, Q., Huo, H.-W. (2011). Algorithms Improving the Storage Efficiency of Deep Packet Inspection. Journal of Software, 22 (1), 149-163. doi: https://doi.org/10.3724/sp.j.1001.2011.03724 